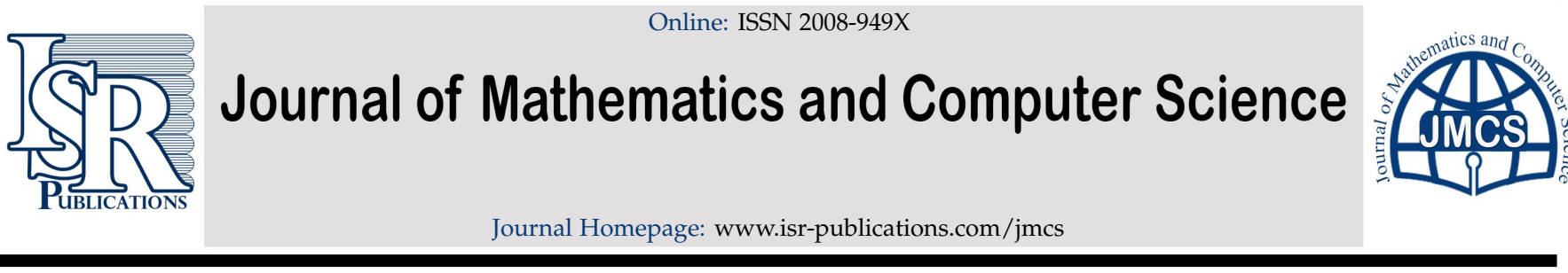

\title{
Numerical solution of Volterra integro-differential equation with delay
}

\author{
Erkan Cimen $^{\mathrm{a}, *}$, Sabahattin Yatar ${ }^{\mathrm{b}}$ \\ ${ }^{a}$ Department of Mathematics, Faculty of Education, Van Yuzuncu Yil University, 65080, Van, Turkey. \\ ${ }^{b}$ Department of Mathematics, Institute of Pure and Applied Sciences, Van Yuzuncu Yil University, 65080, Van, Turkey.
}

\begin{abstract}
We consider an initial value problem for a linear first-order Volterra delay integro-differential equation. We develop a novel difference scheme for the approximate solution of this problem via a finite difference method. The method is based on the fitted difference scheme on a uniform mesh which is achieved by using the method of integral identities which includes the exponential basis functions and applying to interpolate quadrature formulas that contain the remainder term in integral form. Also, the method is proved to be first-order convergent in the discrete maximum norm. Furthermore, a numerical experiment is performed to verify the theoretical results. Finally, the proposed scheme is compared with the implicit Euler scheme.
\end{abstract}

Keywords: Volterra delay integro-differential equation, finite difference method, error estimate.

2010 MSC: 34K28, 65L10, 65L20, 65L70, 65R20.

(C)2020 All rights reserved.

\section{Introduction}

Volterra delay integro-differential equations (VDIDEs) arise widely in mathematical models of biology, medicine, physics phenomena. In particular, the propagation of nervous impulse, population dynamics, polymeric liquids can be modelled by these equations [5, 9, 12, 13, 15, 17].

Motivated by the above works, we consider the following VDIDE in the interval $\overline{\mathrm{I}}=[0, \mathrm{~T}]$ :

$$
\begin{aligned}
& \mathrm{Lu}:=\mathrm{u}^{\prime}(\mathrm{t})+\mathrm{a}(\mathrm{t}) \mathrm{u}(\mathrm{t})+\mathrm{b}(\mathrm{t}) \mathrm{u}(\mathrm{t}-\mathrm{r})=\mathrm{f}(\mathrm{t})+\int_{t-r}^{t} \mathrm{k}(\mathrm{t}, \mathrm{s}) \mathrm{u}(\mathrm{s}) \mathrm{ds}, \mathrm{t} \in \mathrm{I}, \\
& \mathrm{u}(\mathrm{t})=\varphi(t), t \in \mathrm{I}_{0},
\end{aligned}
$$

where $I=(0, T]=\cup_{p=1}^{m} I_{p}, I_{p}=\left\{t: r_{p-1}<t \leqslant r_{p}\right\}, 1 \leqslant p \leqslant m$ and $r_{s}=s r, 0 \leqslant s \leqslant m$ and, $I_{0}=[-r, 0]$ (for simplicity we suppose that $T / r$ is integer; i.e., $T=m r$ ). $a(t) \geqslant \alpha>0, b(t), f(t), \varphi(t)$ and $K(t, s)$ are given sufficiently smooth functions, $r$ is a positive constant large delay. The existence and uniqueness of solution to VDIDEs is discussed in [3,6-8] (see also references cited in them).

\footnotetext{
${ }^{*}$ Corresponding author

Email addresses: cimenerkan@hotmail.com (Erkan Cimen), sabahattin.258@hotmail.com (Sabahattin Yatar)

doi: $10.22436 /$ jmcs.020.03.08
}

Received: 2019-07-28 Revised: 2019-12-05 Accepted: 2019-12-09 
In recent years, various stability analysis and numerical approaches for VDIDEs have been increasingly studied via many methods. For instance, Koto [14] and Rihan et al. [18] studied stability of RungeKutta method for VDIDEs with a constant delay, Zang and Vandewalle [21] proposed an alternative approach method by using the general linear methods with compound quadrature rules for VDIDEs, the dissipativity of $\theta$-methods proposed by Gan [10] the analytical and numerical solution of nonlinear VDIDEs, the stability of linear multistep methods for VDIDEs discussed by Huang [11]. A collocation method with standard software for solving VDIDEs proposed by Shakourifar and Enright in [19]. Zhao et al. [23] proposed some stability results of numerical solution of linear VDIDEs with real coefficients. A Taylor polynomial method for solving VDIDEs presented by Belloura and Bousselsal [4]. Zhao et al. [22] constructed a methodology based on the Sinc collocation technique to approximate pantograph VDIDEs. Abdi et. al. [1] proposed barycentric rational method for solving VDIDEs. Kudu et al. [16] and Yapman et. al. [20] studied finite difference method to approximate solution of singularly perturbed VDIDEs.

In this paper, we develop a novel numerical approach to solve (1.1)-(1.2). Our approach is based on the method of integral identities with the use of interpolating quadrature rules with the weight and remainder terms in integral form. And consequently, the local truncation errors occurs that includes up to first order derivative of the exact solution and thus facilitates examination of the convergence. The remainder of this paper is as follows. In Section 2, we give some important properties of the exact solution of (1.1)-(1.2). We describe the finite difference discretization on uniform mesh in Section 3. We present the error analysis of the proposed scheme and the convergence is proved in the discrete maximum norm in Section 4 . In Section 5, we carry out the numerical experiment which validate the theoretical analysis computationally. Also, we compare our results with the results by obtained classical Euler method. Finally, in the last section, we give some conclusions.

Notation. Throughout the paper, $\mathrm{C}$ will denote a generic positive constant independent of the mesh parameter. Also, some specific fixed constants of this kind are indicated by sub-scripting $C$ and $D .\|g\|_{\infty}$ denotes the continuous maximum norm on the corresponding interval for any continuous function $g(t)$.

\section{Some properties of the problem}

In this section, we give a priori estimates for the exact solution of (1.1)-(1.2), which are used in later sections in the analysis of the approximate solution.

Lemma 2.1. Let $\mathrm{a}, \mathrm{b}, \mathrm{f} \in \mathrm{C}(\overline{\mathrm{I}}), \varphi \in \mathrm{C}\left(\mathrm{I}_{0}\right)$ and $\mathrm{K} \in \mathrm{C}(\overline{\mathrm{I}} \times \overline{\mathrm{I}})$. Then for $\mathrm{u}$ which is the exact solution of the problem (1.1)-(1.2) the following estimates hold:

$$
\begin{array}{r}
\|u\|_{\infty, p} \leqslant C_{p}, 1 \leqslant p \leqslant m, \\
\left\|u^{\prime}\right\|_{\infty, p} \leqslant D_{p}, 1 \leqslant p \leqslant m,
\end{array}
$$

where

and

$$
\begin{aligned}
C_{p} & =\|\varphi\|_{\infty, 0} \delta^{p}+\alpha^{-1} \frac{1-\delta^{p}}{1-\delta}\left(\|f\|_{\infty, p}+\bar{K}\|\varphi\|_{1,0}\right) e^{-\alpha^{-1} \bar{K} T}, 1 \leqslant p \leqslant m, \\
D_{1} & =\left(\|\mathrm{a}\|_{\infty, 1}+\bar{K} T\right) C_{1}+\|b\|_{\infty, 1}\|\varphi\|_{\infty, 0}+\|f\|_{\infty, 1}+\bar{K}\|\varphi\|_{1,0}, \\
D_{p} & =\left(\|a\|_{\infty, p}+\bar{K} T\right) C_{p}+\|b\|_{\infty, p} C_{p-1}+\|f\|_{\infty, p}+\bar{K}\|\varphi\|_{1,0}, 2 \leqslant p \leqslant m,
\end{aligned}
$$

$$
\begin{aligned}
\delta & =\left(1+\alpha^{-1}\|\mathrm{~b}\|_{\infty, p}\right) e^{\alpha^{-1} \overline{\mathrm{K} T}}, \overline{\mathrm{K}}=\max _{(\mathrm{t}, \mathrm{s}) \in \overline{\mathrm{I}} \times \overline{\mathrm{I}}}|\mathrm{K}(\mathrm{t}, \mathrm{s})|, \\
\|\varphi\|_{1,0} & =\int_{-r}^{0}|\varphi(\mathrm{s})| \mathrm{ds} .
\end{aligned}
$$


Proof. Since

$$
\left|\int_{t-r}^{t} K(t, s) u(s) d s\right| \leqslant \bar{K} \int_{t-r}^{t}|u(s)| d s=\bar{K} \begin{cases}\int_{t-r}^{0}|\varphi(s)| d s+\int_{0}^{t}|u(s)| d s, & \text { for } 0<t \leqslant r, \\ \int_{t-r}^{t}|u(s)| d s, & \text { for } t>r,\end{cases}
$$

then we can write

$$
\left|\int_{t-r}^{t} K(t, s) u(s) d s\right| \leqslant \bar{K}\left(\|\varphi\|_{1,0}+\int_{0}^{t}|u(s)| d s\right) .
$$

Now, for $t \in I_{p}$, from (1.1) we have

$$
u(t)=u\left(r_{p-1}\right) e^{-\int_{p-1}^{t} a(\tau) d \tau}+\int_{r_{p-1}}^{t} F(\xi) e^{-\int_{\xi}^{t} a(\tau) d \tau} d \xi
$$

with

$$
F(t)=f(t)-b(t) u(t-r)+\int_{t-r}^{t} K(t, s) u(s) d s
$$

So,

$$
\begin{aligned}
|u(t)| & \leqslant\left|u\left(r_{p-1}\right)\right| e^{-\int_{p-1}^{t} a(\tau) d \tau}+\int_{r_{p-1}}^{t}\left[|f(\xi)|+|b(\xi)||u(\xi-r)|+\int_{\xi-r}^{\xi}|K(\xi, s)||u(s)| d s\right] e^{-\int_{\xi}^{t} a(\tau) d \tau} d \xi, \\
& \leqslant\left|u\left(r_{p-1}\right)\right| e^{-\alpha\left(t-r_{p-1}\right)}+\int_{r_{p-1}}^{t}\left[|f(\xi)|+|b(\xi)||u(\xi-r)|+\int_{\xi-r}^{\xi}|K(\xi, s)||u(s)| d s\right] e^{-\alpha(t-\xi)} d \xi .
\end{aligned}
$$

Then, taking into account the (2.3) inequality in (2.4), we get

$$
\begin{aligned}
\|u\|_{\infty, p} & \leqslant\|u\|_{\infty, p-1}+\alpha^{-1}\left[\|f\|_{\infty, p}+\|b\|_{\infty, p}\|u\|_{\infty, p-1}+\bar{K}\left(\|\varphi\|_{1,0}+\int_{0}^{t}|u(s)| d s\right)\right] \\
& \leqslant\left(1+\alpha^{-1}\|b\|_{\infty, p}\right)\|u\|_{\infty, p-1}+\alpha^{-1}\left[\|f\|_{\infty, p}+\bar{K}\left(\|\varphi\|_{1,0}+\int_{0}^{T}|u(s)| d s\right)\right] .
\end{aligned}
$$

Next, by using the Gronwall's inequality it follows that

$$
\|u\|_{\infty, p} \leqslant\left[\left(1+\alpha^{-1}\|b\|_{\infty, p}\right)\|u\|_{\infty, p-1}+\alpha^{-1}\left(\|f\|_{\infty, p}+\bar{K}\|\varphi\|_{1,0}\right)\right] e^{\alpha^{-1} \bar{K} T} .
$$

From here, the following first order difference inequality follows

$$
v_{p} \leqslant q v_{p-1}+\lambda
$$

with

$$
v_{p}=\|u\|_{\infty, p}, q=\left(1+\alpha^{-1}\|b\|_{\infty, p}\right) e^{\alpha^{-1} \bar{K} T}, \quad \lambda=\alpha^{-1}\left(\|f\|_{\infty, p}+\bar{K}\|\varphi\|_{1,0}\right) e^{\alpha^{-1} \bar{K} T},
$$

which yields the estimate

$$
v_{p} \leqslant v_{0} q^{p}+\lambda \sum_{s=1}^{p} q^{p-s}
$$

and so we arrive at (2.1). The proof of (2.2) is by induction in p. From (1.1) and (2.3), we have

$$
\left|u^{\prime}(t)\right| \leqslant|a(t)||u(t)|+|b(t)||u(t-r)|+|f(t)|+\bar{k}\left(\|\varphi\|_{1,0}+\int_{0}^{t}|u(s)| d s\right) .
$$


Now, for $p=1\left(t \in I_{1}\right)$, we have

$$
\left|\mathrm{u}^{\prime}(\mathrm{t})\right| \leqslant\|\mathrm{a}\|_{\infty, 1}\|\mathrm{u}\|_{\infty, 1}+\|\mathrm{b}\|_{\infty, 1}\|\varphi\|_{\infty, 0}+\|\mathrm{f}\|_{\infty, 1}+\overline{\mathrm{K}}\left(\|\varphi\|_{1,0}+\|\mathrm{u}\|_{\infty, 1} \mathrm{~T}\right) \equiv \mathrm{D}_{1} .
$$

Let the inequality (2.2) be true for $p=k$. That is

$$
D_{k}=\left(\|\mathrm{a}\|_{\infty, k}+\overline{\mathrm{K}} T\right) \mathrm{C}_{\mathrm{k}}+\|\mathrm{b}\|_{\infty, \mathrm{k}} \mathrm{C}_{\mathrm{k}-1}+\|\mathrm{f}\|_{\infty, \mathrm{k}}+\overline{\mathrm{K}}\|\varphi\|_{1,0} .
$$

For $t \in I_{k+1}$ because of (2.5) we get

$$
\left|u^{\prime}(t)\right| \leqslant\|a\|_{\infty, k+1}\|u\|_{\infty, k+1}+\|b\|_{\infty, k+1}\|u\|_{\infty, k}+\|f\|_{\infty, k+1}+\bar{K}\left(\|\varphi\|_{1,0}+\|u\|_{\infty, k+1} T\right)
$$

and hence the inequality (2.2) hold for $p=k+1$.

\section{Construction of the difference scheme}

Let $\omega_{\mathrm{N}_{0}}$ be a uniform mesh on $\overline{\mathrm{I}}$ :

$$
\omega_{N_{0}}=\left\{t_{i}=i h, i=1,2, \ldots, N_{0}, h=T / N_{0}=r / N\right\},
$$

which contains by $\mathrm{N}$ mesh point at each subinterval $\mathrm{I}_{\mathrm{p}}(1 \leqslant \mathrm{p} \leqslant \mathrm{m})$ :

$$
\omega_{N_{p}}=\left\{t_{i}:(p-1) N+1 \leqslant i \leqslant p N, 1 \leqslant p \leqslant m\right\}
$$

and consequently

$$
\omega_{\mathrm{N}_{0}}=\cup_{p=1}^{m} \omega_{\mathrm{N}_{\mathrm{p}}} .
$$

For any mesh function $g(t)$, we use $g_{i}=g\left(t_{i}\right)$ and moreover $y_{i}$ denotes an approximation of $u(t)$ at $t_{i}$ and

$$
g_{\bar{t}, i}=\left(g_{i}-g_{i-1}\right) / h,\|g\|_{\infty, p}=\|g\|_{\infty, \omega_{N, p}}:=\max _{1 \leqslant i \leqslant N}\left|g_{i}\right| .
$$

For the difference approximation the problem (1.1), we are using the following identity

$$
h^{-1} \int_{t_{i-1}}^{t_{i}} L u(t) \psi_{i}(t) d t=h^{-1} \int_{t_{i-1}}^{t_{i}}\left[f(t)+\int_{t-r}^{t} K(t, s) u(s) d s\right] \psi_{i}(t) d t, 1 \leqslant i \leqslant N_{0},
$$

with basis function

$$
\psi_{i}(t)=e^{-\int_{t}^{t_{i}} a(\tau) d \tau}, t_{i-1} \leqslant t \leqslant t_{i},
$$

which is the solution of the following problem

$$
-\psi_{i}^{\prime}(t)+a(t) \psi_{i}(t)=0, t_{i-1}<t \leqslant t_{i}, \psi_{i}\left(t_{i}\right)=1 .
$$

The relation (3.1) is rewritten as

$$
\begin{aligned}
& h^{-1} \int_{t_{i-1}}^{t_{i}} u^{\prime}(t) \psi_{i}(t) d t+h^{-1} \int_{t_{i-1}}^{t_{i}} a(t) u(t) \psi_{i}(t) d t+h^{-1} \int_{t_{i-1}}^{t_{i}} b(t) u(t-r) \psi_{i}(t) d t \\
& =h^{-1} \int_{t_{i-1}}^{t_{i}} f(t) \psi_{i}(t) d t+h^{-1} \int_{t_{i-1}}^{t_{i}}\left[\int_{t-r}^{t} K(t, s) u(s) d s\right] \psi_{i}(t) d t .
\end{aligned}
$$

Firstly, using the formulas (2.1) and (2.2) from [2] on each interval $\left(t_{i-1}, t_{i}\right)$ taking into account the left hand side Eq. (3.3) we have

$$
h^{-1} \int_{t_{i-1}}^{t_{i}} u^{\prime}(t) \psi_{i}(t) d t+h^{-1} \int_{t_{i-1}}^{t_{i}} a(t) u(t) \psi_{i}(t) d t+h^{-1} \int_{t_{i-1}}^{t_{i}} b(t) u(t-r) \psi_{i}(t) d t
$$




$$
=A_{i} u_{\bar{t}, i}+B_{i} u_{\bar{t}, i-N}+C_{i} u_{i}+D_{i} u_{i-N}+R_{i}^{(1)},
$$

where

$$
\begin{aligned}
A_{i} & =h^{-1} \int_{t_{i-1}}^{t_{i}} \psi_{i}(t) d t+h^{-1} \int_{t_{i-1}}^{t_{i}}\left(t-t_{i}\right) a(t) \psi_{i}(t) d t, \\
B_{i} & =h^{-1} \int_{t_{i-1}}^{t_{i}}\left(t-t_{i}\right) b(t) \psi_{i}(t) d t \\
C_{i} & =h^{-1} \int_{t_{i-1}}^{t_{i}} a(t) \psi_{i}(t) d t \\
D_{i} & =h^{-1} \int_{t_{i-1}}^{t_{i}} b(t) \psi_{i}(t) d t \\
R_{i}^{(1)} & =h^{-1} \int_{t_{i-1}}^{t_{i}} d t b(t) \psi_{i}(t) \int_{t_{i-1}}^{t_{i}} u^{\prime}(s-r)\left[T_{0}(t-s)-h^{-1}\left(t-t_{i-1}\right)\right] d s .
\end{aligned}
$$

Secondly, for the integral term from the right hand side Eq. (3.3), after applying the appropriate quadrature rules, we have

$$
\begin{aligned}
h^{-1} \int_{t_{i-1}}^{t_{i}}\left[\int_{t-r}^{t} K(t, s) u(s) d s\right] \psi_{i}(t) d t & =h^{-1} \int_{t_{i-1}}^{t_{i}} \psi_{i}(t) d t \int_{t_{i-\frac{1}{2}}-r}^{t_{i-\frac{1}{2}}} K\left(t_{i-\frac{1}{2}}, s\right) u(s) d s+R_{i}^{(2)} \\
& =h^{-1} \int_{t_{i-1}}^{t_{i}} \psi_{i}(t) d t h \sum_{j=i-N}^{i-1} K\left(t_{i-\frac{1}{2}}, s_{j}\right) u_{j}+R_{i}^{(2)}+R_{i}^{(3)},
\end{aligned}
$$

where

$$
\begin{aligned}
& R_{i}^{(2)}=h^{-1} \int_{t_{i-1}}^{t_{i}} \psi_{i}(t) d t \int_{t_{i-1}}^{t_{i}} \frac{d}{d t}\left(\int_{\xi-r}^{\xi} K(\xi, s) u(s) d s\right)\left[T_{0}(t-\xi)-T_{0}\left(t_{i-\frac{1}{2}}-\xi\right)\right] d \xi, \\
& R_{i}^{(3)}=\sum_{j=i-N}^{i-1} \int_{t_{j-\frac{1}{2}}}^{t_{j+\frac{1}{2}}}\left(t_{j+\frac{1}{2}}-\xi-h T_{0}\left(t_{j}-\xi\right)\right) \frac{d}{d s}\left(K\left(t_{i-\frac{1}{2}}, s\right) u(s)\right) d s,
\end{aligned}
$$

and $\mathrm{T}_{0}(\mathrm{t})=1, \mathrm{t}>0 ; \mathrm{T}_{0}(\mathrm{t})=0, \mathrm{t} \leqslant 0$.

Hereby, we write the exact relation for $u\left(t_{i}\right)$ :

$$
\ell u_{i} \equiv A_{i} u_{\bar{t}, i}+B_{i} u_{\bar{t}, i-N}+C_{i} u_{i}+D_{i} u_{i-N}=F_{i}+E_{i} h \sum_{j=i-N}^{i-1} k_{i-\frac{1}{2}, j} u_{j}+R_{i}, 1 \leqslant i \leqslant N_{0}
$$

with

$$
E_{i}=h^{-1} \int_{t_{i-1}}^{t_{i}} \psi_{i}(t) d t, \quad F_{i}=h^{-1} \int_{t_{i-1}}^{t_{i}} f(t) \psi_{i}(t) d t, \quad R_{i}=R_{i}^{(1)}+R_{i}^{(2)}+R_{i}^{(3)}
$$

where $A_{i}, B_{i}, C_{i}, D_{i}$ and $R_{i}^{(k)}(k=1,2,3)$ are determined by (3.4)-(3.7) and (3.8)-(3.10), respectively. By virtue of (3.11) we suggest the following difference scheme for approximating (1.1)-(1.2):

$$
\begin{aligned}
\ell y_{i} & \equiv A_{i} y_{\bar{t}, i}+B_{i} y_{\bar{t}, i-N}+C_{i} y_{i}+D_{i} y_{i-N}=F_{i}+E_{i} h \sum_{j=i-N}^{i-1} K_{i-\frac{1}{2}, j} y_{j}, 1 \leqslant i \leqslant N_{0}, \\
y_{i} & =\varphi_{i},-N \leqslant i \leqslant 0 .
\end{aligned}
$$


We also propose another difference scheme that can be easily obtained using the implicit Euler method and appropriate quadrature rules:

$$
\begin{aligned}
y_{\bar{t}, i}+a_{i} y_{i}+b_{i} y_{i-N} & =f_{i}+h \sum_{j=i-N}^{i-1} k_{i-\frac{1}{2}, j} y_{j}, 1 \leqslant i \leqslant N_{0}, \\
y_{i} & =\varphi_{i},-N \leqslant i \leqslant 0 .
\end{aligned}
$$

\section{Error analysis}

In order to investigate the convergence of this method, note that the error function $z_{i}=y_{i}-u_{i}$, $0 \leqslant i \leqslant N_{0}$ is the solution of the following discrete problem

$$
\begin{aligned}
\ell z_{i} & =R_{i}, 1 \leqslant i \leqslant N_{0}, \\
z_{i} & =0,-N_{0} \leqslant i \leqslant 0,
\end{aligned}
$$

where the truncation error $R_{i}$ is given by (3.12).

Lemma 4.1. If $\mathrm{a}, \mathrm{b}, \mathrm{f} \in \mathrm{C}(\overline{\mathrm{I}}), \varphi \in \mathrm{C}\left(\mathrm{I}_{0}\right)$ and $\mathrm{K} \in \mathrm{C}^{1}(\overline{\mathrm{I}} \times \overline{\mathrm{I}})$, then for the truncation error $\mathrm{R}_{\mathrm{i}}$ we have

$$
\|\mathrm{R}\|_{\infty, p} \leqslant \mathrm{Ch}, 1 \leqslant \mathrm{p} \leqslant \mathrm{m} .
$$

Proof. From (3.8), we write

$$
\left|R_{i}^{(1)}\right| \leqslant C h^{-1} \int_{t_{i-1}}^{t_{i}} d t|b(t)| \psi_{i}(t) \int_{t_{i-1}}^{t_{i}}\left|u^{\prime}(\xi-r)\right| d \xi .
$$

By virtue of Lemma 2.1 and $0<\psi_{i}(t) \leqslant 1$

$$
\left|R_{i}^{(1)}\right| \leqslant C h .
$$

For the estimate $R_{i}^{(2)}$, from (3.9) we have

$$
\left|R_{i}^{(2)}\right| \leqslant C h^{-1} \int_{t_{i-1}}^{t_{i}} d t \psi_{i}(t) \int_{t_{i-1}}^{t_{i}}\left[\left|\frac{\partial}{\partial \xi} K(\xi, s)\right||u(s)|+|K(\xi, \xi)||u(\xi)|+|K(\xi, \xi-r)||u(\xi)|\right] d \xi
$$

and in view of Lemma 2.1, $\left|\frac{\partial}{\partial t} K(t, s)\right| \leqslant C$, and $0<\psi_{i}(t) \leqslant 1$,

$$
\left|R_{i}^{(2)}\right| \leqslant C h \text {. }
$$

Now, for the $R_{i}^{(3)}$, from (3.10) we have

$$
\left|R_{i}^{(3)}\right| \leqslant C h \sum_{j=i-N}^{i-1} \int_{t_{j-\frac{1}{2}}}^{t_{j+\frac{1}{2}}}\left[\left|\frac{\partial}{\partial s} K\left(t_{i-\frac{1}{2}}, s\right)\right||u(s)|+\left|K\left(t_{i-\frac{1}{2}}, s\right)\right|\left|u^{\prime}(s)\right|\right] d s
$$

and in view of Lemma 2.1 and $\left|\frac{\partial}{\partial s} K(t, s)\right| \leqslant C$

$$
\left|R_{i}^{(3)}\right| \leqslant C h \sum_{j=i-N}^{i-1}\left(t_{j+\frac{1}{2}}-t_{j-\frac{1}{2}}\right)=\mathrm{Ch}^{2} \mathrm{~N} .
$$

Lemma 4.2. Let $z_{i}$ be the solution of (4.1)-(4.2) holds true. Then

$$
\|z\|_{\infty, p} \leqslant C \sum_{k=1}^{p}\|R\|_{\infty, k}, 1 \leqslant p \leqslant m
$$


Proof. Since

$$
\left|h \sum_{j=i-N}^{i-1} \kappa_{i-\frac{1}{2}, j} z_{j}\right| \leqslant h \bar{K} \sum_{j=i-N}^{i-1}\left|z_{j}\right| \leqslant \begin{cases}h \bar{K} \sum_{j=i-N}^{0}\left|\varphi_{j}\right|, & i=1, \\ h \bar{K} \sum_{j=i-N}^{0}\left|\varphi_{j}\right|+h \bar{K} \sum_{j=1}^{i-1}\left|z_{j}\right|, & 1<i \leqslant N, \\ h \bar{K} \sum_{j=i-N}^{i-1}\left|z_{j}\right|, & i>N,\end{cases}
$$

then it is not hard to see that

$$
\left|f_{i}+h \sum_{j=i-N}^{i-1} K_{i-\frac{1}{2}, j} z_{j}\right| \leqslant\|f\|_{\infty, p}+\bar{K}\|\varphi\|_{1,0}+h \bar{K} \sum_{j=1}^{i}\left|z_{j-1}\right| .
$$

From the solution (4.1)-(4.2)

$$
\|z\|_{\infty, p} \leqslant \alpha^{-1} \sum_{k=1}^{p}\|R\|_{\infty, k} Q_{p-k}, \quad 1 \leqslant p \leqslant m
$$

where

$$
Q_{p-k}= \begin{cases}1, & \text { for } k=p \\ \prod_{s=k+1}^{p}\left(1+\alpha^{-1}\left(\|\mathrm{a}\|_{\infty, I_{s}}+\|c\|_{\infty, I_{s}}\right)\right), & \text { for } 0 \leqslant k \leqslant p-1 .\end{cases}
$$

Hence, the proof is followed easily by induction in $p$.

Combining Lemmas 4.1 and 4.2 gives us the following convergence result.

Theorem 4.3. If $\mathrm{u}$ be the solution of (1.1)-(1.2) and $\mathrm{y}$ the solution of (3.1)-(3.2), then the following estimate holds true:

$$
\|y-u\|_{\infty, \omega_{N_{0}}} \leqslant C h
$$

\section{Numerical results}

In this section, we present numerical experiment for the problem (1.1)-(1.2) to verify the theoretical results. We obtain numerical results by applying the new difference scheme (3.13)-(3.14) to the particular problem. Also, we present numerical results obtained by using implicit Euler method in (3.15)-(3.16). In addition, we compare both methods with respect to maximum pointwise errors.

Example 1. We consider the test problem:

$$
u^{\prime}(t)+2 u(t)-u(t-1)=e^{t-1}-2 t+\int_{t-1}^{t}(t-s) u(s) d s, 0<t \leqslant 2,
$$

subject to the interval condition

$$
u(t)=e^{t},-1 \leqslant t \leqslant 0,
$$

the exact solution of the problem is given by

$$
u(t)= \begin{cases}e^{-t}, & t \in(0,1], \\ \frac{4}{\sqrt{5}}\left[\delta^{-} e^{\delta^{-}(t-1)}-\delta^{+} e^{\delta^{+}(t-1)}\right]+\frac{1}{2} e^{t-1}+\left(\frac{9}{2}+e^{-1}-t\right) e^{1-t}, & t \in(1,2],\end{cases}
$$

where

$$
\delta^{ \pm}=(-1 \pm \sqrt{5}) / 2
$$

We define the exact error $e_{i}^{N}$ and the computed maximum pointwise error $e^{N}$ for any $\mathrm{N}$ as follows:

$$
e_{i}^{N}=\left|y_{i}-u_{i}\right|, e^{N}=\max _{0 \leqslant i \leqslant N} e_{i}^{N},
$$

where $y_{i}$ is the numerical approximation to exact value $u_{i}$ for the nodes $t_{i}$. The computational results of the test problem obtained by using both present method (PM) and implicit Euler method (EM) are given in the Tables 1-3. 
Table 1: The numerical results for the test problem on $(0,2](\mathrm{PM})$.

\begin{tabular}{cccccc}
\hline $\mathrm{t}_{i}$ & $\mathrm{u}_{i}$ & $\mathrm{y}_{i}(\mathrm{~N}=64)$ & $e_{i}^{64}$ & $\mathrm{y}_{i}(\mathrm{~N}=128)$ & $e_{i}^{128}$ \\
\hline 0.125 & 0.8824969 & 0.8824977 & $8.158 \mathrm{E}-7$ & 0.8824971 & $2.040 \mathrm{E}-7$ \\
0.250 & 0.7788008 & 0.7788030 & $2.225 \mathrm{E}-6$ & 0.7788013 & $5.563 \mathrm{E}-7$ \\
0.375 & 0.6872893 & 0.6872935 & $4.251 \mathrm{E}-6$ & 0.6872903 & $1.063 \mathrm{E}-6$ \\
0.500 & 0.6065307 & 0.6065376 & $6.921 \mathrm{E}-6$ & 0.6065324 & $1.731 \mathrm{E}-6$ \\
0.625 & 0.5352614 & 0.5352717 & $1.027 \mathrm{E}-5$ & 0.5352640 & $2.568 \mathrm{E}-6$ \\
0.750 & 0.4723666 & 0.4723809 & $1.434 \mathrm{E}-5$ & 0.4723701 & $3.584 \mathrm{E}-6$ \\
0.875 & 0.4168620 & 0.4168812 & $1.918 \mathrm{E}-5$ & 0.4168668 & $4.794 \mathrm{E}-6$ \\
1.000 & 0.3678794 & 0.3679043 & $2.485 \mathrm{E}-5$ & 0.3678857 & $6.214 \mathrm{E}-6$ \\
1.125 & 0.3108644 & 0.3108857 & $2.128 \mathrm{E}-5$ & 0.3108697 & $5.319 \mathrm{E}-6$ \\
1.250 & 0.2378586 & 0.2378772 & $1.859 \mathrm{E}-5$ & 0.2378632 & $4.648 \mathrm{E}-6$ \\
1.375 & 0.1563964 & 0.1564131 & $1.672 \mathrm{E}-5$ & 0.1564006 & $4.179 \mathrm{E}-6$ \\
1.500 & 0.0723224 & 0.0723380 & $1.559 \mathrm{E}-5$ & 0.0723263 & $3.897 \mathrm{E}-6$ \\
1.625 & -0.0097872 & -0.0097720 & $1.514 \mathrm{E}-5$ & -0.0097834 & $3.785 \mathrm{E}-6$ \\
1.750 & -0.0862851 & -0.0862697 & $1.531 \mathrm{E}-5$ & -0.0862812 & $3.828 \mathrm{E}-6$ \\
1.875 & -0.1541774 & -0.1541614 & $1.604 \mathrm{E}-5$ & -0.1541734 & $4.009 \mathrm{E}-6$ \\
2.000 & -0.2108996 & -0.2108823 & $1.726 \mathrm{E}-5$ & -0.2108953 & $4.314 \mathrm{E}-6$ \\
\hline
\end{tabular}

Table 2: The numerical results for the test problem on $(0,2](\mathrm{EM})$.

\begin{tabular}{cccccc}
\hline $\mathrm{t}_{i}$ & $\mathrm{u}_{i}$ & $\mathrm{y}_{i}(\mathrm{~N}=64)$ & $e_{i}^{64}$ & $\mathrm{y}_{i}(\mathrm{~N}=128)$ & $e_{i}^{128}$ \\
\hline 0.125 & 0.8824969 & 0.8830591 & $5.622 \mathrm{E}-3$ & 0.8827802 & $2.833 \mathrm{E}-4$ \\
0.250 & 0.7788008 & 0.7797059 & $9.051 \mathrm{E}-4$ & 0.7792562 & $4.554 \mathrm{E}-4$ \\
0.375 & 0.6872893 & 0.6884010 & $1.112 \mathrm{E}-3$ & 0.6878477 & $5.584 \mathrm{E}-4$ \\
0.500 & 0.6065307 & 0.6077771 & $1.246 \mathrm{E}-3$ & 0.6071556 & $6.249 \mathrm{E}-4$ \\
0.625 & 0.5352614 & 0.5366208 & $1.359 \mathrm{E}-3$ & 0.5359418 & $6.804 \mathrm{E}-4$ \\
0.750 & 0.4723666 & 0.4738566 & $1.490 \mathrm{E}-3$ & 0.4731112 & $7.446 \mathrm{E}-4$ \\
0.875 & 0.4168620 & 0.4185319 & $1.670 \mathrm{E}-3$ & 0.4176955 & $8.336 \mathrm{E}-4$ \\
1.000 & 0.3678794 & 0.3698046 & $1.925 \mathrm{E}-3$ & 0.3688397 & $9.603 \mathrm{E}-4$ \\
1.125 & 0.3108644 & 0.3116622 & $7.978 \mathrm{E}-4$ & 0.3112550 & $3.906 \mathrm{E}-4$ \\
1.250 & 0.2378586 & 0.2382602 & $4.016 \mathrm{E}-4$ & 0.2380500 & $1.914 \mathrm{E}-4$ \\
1.375 & 0.1563964 & 0.1568536 & $4.572 \mathrm{E}-4$ & 0.1566167 & $2.203 \mathrm{E}-4$ \\
1.500 & 0.0723224 & 0.0731035 & $7.811 \mathrm{E}-4$ & 0.0727067 & $3.843 \mathrm{E}-4$ \\
1.625 & -0.0097872 & -0.0085294 & $1.258 \mathrm{E}-3$ & -0.0091625 & $6.247 \mathrm{E}-4$ \\
1.750 & -0.0862851 & -0.0844663 & $1.819 \mathrm{E}-3$ & -0.0853781 & $9.070 \mathrm{E}-4$ \\
1.875 & -0.1541774 & -0.1517501 & $2.427 \mathrm{E}-3$ & -0.1529648 & $1.213 \mathrm{E}-3$ \\
2.000 & -0.2108996 & -0.2078312 & $3.068 \mathrm{E}-3$ & -0.2093656 & $1.534 \mathrm{E}-3$ \\
\hline
\end{tabular}

Table 3: Comparison of $e^{\mathrm{N}}$ both methods on $(0,2]$.

\begin{tabular}{cccccc}
\hline $\mathrm{N}$ & $e^{\mathrm{N}}(\mathrm{EM})$ & $e^{\mathrm{N}}(\mathrm{PM})$ & $\mathrm{N}$ & $e^{\mathrm{N}}(\mathrm{EM})$ & $e^{\mathrm{N}}(\mathrm{PM})$ \\
\hline 32 & $6.138 \mathrm{E}-3$ & $9.938 \mathrm{E}-5$ & 256 & $7.670 \mathrm{E}-4$ & $1.553 \mathrm{E}-6$ \\
64 & $3.068 \mathrm{E}-3$ & $2.485 \mathrm{E}-5$ & 512 & $3.835 \mathrm{E}-4$ & $3.884 \mathrm{E}-7$ \\
128 & $1.534 \mathrm{E}-3$ & $6.214 \mathrm{E}-6$ & 1024 & $1.917 \mathrm{E}-4$ & $9.709 \mathrm{E}-8$ \\
\hline
\end{tabular}

\section{Acknowledgment}

We thank the editor(s) and the referee(s) for their favorable comments. 


\section{References}

[1] A. Abdi, J.-P. Berrut, S. A. Hosseini, The linear barycentric rational method for a class of delay Volterra integro-differential equations, J. Sci. Comput., 75 (2018), 1757-1775. 1

[2] G. M. Amiraliyev, Y. D. Mamedov, Difference schemes on the uniform mesh for singular perturbed pseudo-parabolic equations, Turkish J. Math., 19 (1995), 207-222. 3

[3] A. Bellen, M. Zennaro, Numerical methods for delay differential equations, Oxford University Press, New York, (2003). 1

[4] A. Bellour, M. Bousselsal, Numerical solution of delay integro-differential equations by using Taylor collocation method, Math. Methods Appl. Sci., 37 (2014), 1491-1506. 1

[5] G. A. Bocharov, F. A. Rihan, Numerical modelling in biosciences with delay differential equations, J. Comput. Appl. Math., 125 (2000), 183-199. 1

[6] H. Brunner, Collocation methods for Volterra integral and related functional differential equations, Cambridge University Press, Cambridge, (2004). 1

[7] H. Brunner, W. Zhang, Primary discontinuities in solutions for delay integro-differential equations, Methods Appl. Anal., 6 (1999), 525-533.

[8] V. A. Caus, Delay integral equations, An. Univ. Oradea Fasc. Mat., 9 (2002), 109-112. 1

[9] J. M. Cushing, Integrodifferential equations and delay models in population dynamics, Springer-Verlag, New York, (1977). 1

[10] S. Gan, Dissipativity of $\theta$-methods for nonlinear Volterra delay-integro-differential equations, J. Comput. Appl. Math., 206 (2007), 898-907. 1

[11] C. M. Huang, Stability of linear multistep methods for delay integro-differential equations, Comput. Math. Appl., 55 (2008), 2830-2838. 1

[12] A. J. Jerri, Introduction to integral equations with applications, Wiley-Interscience, New York, (1999). 1

[13] V. Kolmanovskii, A. Myshkis, Introduction to the theory and applications of functional differential equations, Kluwer Academic Publishers, Dordrecht, (1999). 1

[14] T. Koto, Stability of Runge-Kutta methods for delay integro-differential equations, J. Comput. Appl. Math., 145 (2002), 483-492. 1

[15] K. Kuang, Delay differential equations with applications in population dynamics, Academic Press, Boston, (1993). 1

[16] M. Kudu, I. Amirali, G. M. Amiraliyev, A finite-difference method for a singularly perturbed delay integro-differential equation, J. Comput. Appl. Math., 308 (2016), 379-390. 1

[17] P. Markowich, M. Renardy, A nonlinear Volterra integro-differential equation describing the stretching of polymeric liquids, SIAM J. Math. Anal., 14 (1983), 66-97. 1

[18] F. A. Rihan, E. H. Doha, M. I. Hassan, N. M. Kamel, Numerical treatments for Volterra delay integro-differential equations, Comput. Methods Appl. Math., 9 (2009), 292-308. 1

[19] M. Shakourifar, W. Enright, Superconvergent interpolants for collocation methods applied to Volterra integro-differential equations with delay, BIT Numer. Math., 52 (2012), 725-740. 1

[20] Ö. Yapman, G. M. Amiraliyev, I. Amirali, Convergence analysis of fitted numerical method for a singularly perturbed nonlinear Volterra integro-differential equation with delay, J. Comput. Appl. Math., 355 (2019), 301-309. 1

[21] C. J. Zhang, S. Vandewalle, Stability analysis of Volterra delay-integro-differential equations and their backward differentiation time discretization, J. Comput. Appl. Math., 164/165 (2004), 797-814. 1

[22] J. J. Zhao, Y. Cao, Y. Xu, Sinc numerical solution for pantograph Volterra delay-integro-differential equation, Int. J. Comput. Math., 94 (2017), 853-865. 1

[23] J. J. Zhao, Y. Fan, Y. Xu, Delay-dependent stability analysis of symmetric boundary value methods for linear delay integrodifferential equations, Numer. Algorithms, 65 (2014), 125-151. 1 